\title{
FAST AUTONOMOUS FLIGHT WITH MICRO UNMANNED VEHICLES
}

\author{
Yash Mulgaonkar*, Anurag Makineni, Kartik Mohta, Camillo J. Taylor, Vijay Kumar \\ GRASP Laboratory, University of Pennsylvania, Philadelphia, Pennsylvania, USA
}

\begin{abstract}
The last decade has seen a proliferation of Micro Aerial Vehicles (MAVs), particularly multirotor vehicles. Indeed quadrotor and hexrotor platforms can be bought off the shelf, and the basic technology has been commoditized. However, high-speed agile flight in GPS-denied environments remains a challenge. This is because the weight of the onboard sensors and processors that are required for autonomy increases the size and inertia of the vehicle, and decreases its maneuverability. In this paper, we present the FALCON, a quadrotor MAV) capable of autonomous high speed flight in complex environments using onboard sensors for state estimation. We discuss the design considerations required for high speed flight and the energetics of quadrotor platforms, and provide specifics on the FALCON design and its performance.
\end{abstract}

\section{INTRODUCTION}

The last decade has seen rapid progress in the use of micro aerial vehicles (MAVs) as a research platform. Rotorcrafts constitute an important class of MAVs. They are of particular interest due to their ability to hover in place, and quickly transitioning from hover to forward flight and back. They include conventional and co-axial helicopters [1, quadrotors 2, hexarotors and ducted fans 3 . Of these, quadrotors have proved to be the most commonly used aerial platform in robotics research labs. In this class, the Hummingbird and Pelican quadrotors manufactured by Ascending Technologies, GmbH 4, with a tip-to-tip footprint of under $75 \mathrm{~cm}$ and weighing less than $1 \mathrm{~kg}$ have proven to be remarkably capable and robust platforms as shown in [5] [6]. The basic design for a quadrotor is simple and the cost of the basic components is low. Indeed the number of drone companies using this design today shows that the barrier to designing and manufacturing quadrotor platforms is low.

Rotorcrafts are used today in a variety of applications including inspection, photography, and data collection in the entertainment, construction and insurance industry. Quadrotors have been proposed for surveillance and search and rescue missions in confined indoor and outdoor environments due to their small size, agility, and ability to hover. In such missions, it is essential that the MAV is capable of autonomous flight to minimize operator workload. Their capability to execute aggressive trajectories [6] and maneuver in $3 \mathrm{D}$ constrained environments, autonomously generate $3-\mathrm{D}$ maps of unknown environments [7, autonomously build 3-D structures using grippers [8, and form swarms of robots flying in formation [9] opens the door to a wide variety of new applications for this technology.

This paper presents the design considerations for a quadrotor platform capable of high-speed agile flight in GPSdenied environments. We use scaling laws to illustrate the benefits of small size and empirical results to show the value of developing light weight platforms. Finally, we present the detailed design of the FALCON platform, which has an un-

978-1-940470-02-3/HH2016/\$25C2016TRF precedented thrust-to-weight ratio of 2.5 and a maximum speed of $20 \mathrm{~m} / \mathrm{s}$.

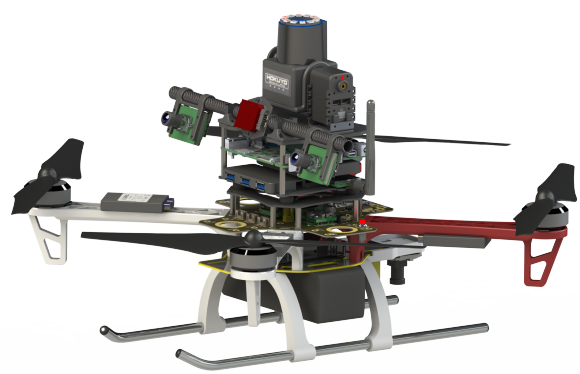

Figure 1: CAD model of the FALCON robot

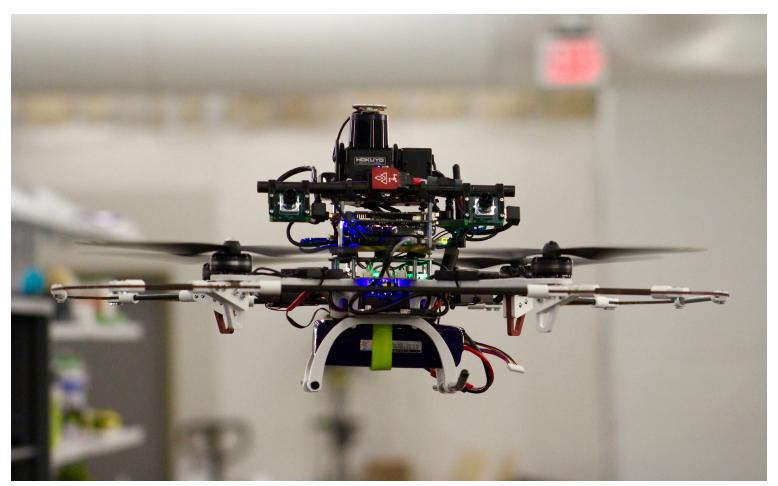

Figure 2: The FALCON robot

\section{MECHANICS}

Most MAVs are equipped with sensors like MEMS IMUs, GPS, cameras, and laser scanners etc. Robust state estimation for autonomous flight in GPS-denied environments frequently requires multiple sensors such as laser scanners 10, monocular cameras 11], stereo cameras 12, and RGB-D sensors 13. Sensors increase the payload that must be carried, increasing the size of the MAV and its battery.

Scaling up in size to accommodate heavier payloads has a dramatic effect on aspects such as inertia of the robot and flight time 14. As shown in our previous work 15, the blade tip speed, $v_{b}$, for small quadrotors scales with the square root of the characteristic length $L$. This is often called the Froude scaling paradigm in which the Froude number, given by

$$
F r=\frac{v_{b}^{2}}{L g}=\frac{\omega^{2} r^{2}}{L g}
$$

is a constant, where $r$ is the rotor radius and $g$ is the acceleration due to gravity. Assuming that the rotor radius $r$ scales linearly with the characteristic length of the robot, $L$, and observing that

$$
\omega=\frac{v_{b}}{r},
$$

Solid-State Sensors, Actuators and Microsystems Workshop Hilton Head Island, South Carolina, June 5-9, 2016 
and the inertia of the robot, $I$, scales inversely with the size of the robot,

$$
I=\frac{1}{L^{5}}
$$

we can show that the maximum angular acceleration $\alpha$ [15] scales as:

$$
\alpha \sim \frac{1}{L}
$$

This analysis suggests that by reducing the size of the vehicle by a factor of two, the vehicle's maximum angular acceleration doubles.

A simple rigid body dynamics model can be used to analyze the maneuverability of a quadrotor. Figure 3 illustrates the effect of improved angular acceleration on maneuverability. Increasing the angular acceleration decreases the turning radius, and this increase is particularly significant at high speeds. The same model shows that the distance taken for the vehicle to come to rest at a hover position decreases similarly with higher angular accelerations.

In [14, we also show that quadrotors for which $L$ is roughly 1 meter consumes approximately $200 \mathrm{~W} / \mathrm{kg}$. If we are designing a vehicle for a prescribed flight time, increasing the payload of the vehicle results in an increase in power consumption which also increases the weight of the battery. Thus the weight penalty increases substantially. Unfortunately sensors such as laser scanners are heavy and can weigh hundreds of grams. And yet these are necessary for reliable detection of obstacles since they provide estimates of distances to most obstacles in the environment under a wide range of operating conditions.

FALCON represents our effort to design a quadrotor platform that is capable of autonomous navigation and yet is highly maneuverable. While the presence of heavy sensors drives up the payload, the use of appropriate motors and propellors maximizes the thrust to weight ratio. In this paper, we present the $3 \mathrm{~kg}$ FALCON which is equipped with an IMU, a gimballed laser scanner, stereo cameras, laser altimeter, and magnetometer. All the computation for the FALCON is performed onboard on an Intel NUC computer with a 5 th generation i7 processor.

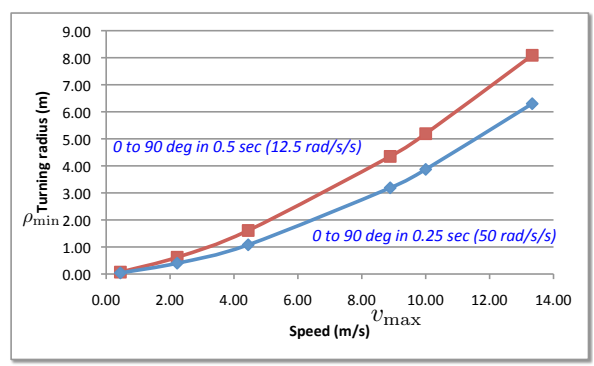

Figure 3: Maneuverability at high speeds. The minimum turning radius is shown for two different limits on angular acceleration (governed by the inertia and the thrust) for a thrust to weight ratio of 2.0. The minimum turning radius is also equal to the stopping distance for maneuvers in which the robot must brake from an initial speed of $v_{\max }$ to come to a stop.

\section{AUTONOMY}

There are several elements essential for fast autonomous flight. Perhaps the most important element is state estimation, the reliable estimation of position and orientation and their derivatives at high rates even when GPS is not available. Next, we require nonlinear controllers that are able to control the position and attitude of the vehicle even at high speeds, at states that are not close to the hover state. We also need the ability to create dense, 3-D maps of the environment allowing the vehicle to detect obstacles. Dense mapping allows motion planning in three dimensions allowing the vehicle to safely navigate to the goal. Each of these elements is discussed next.

\section{State Estimation}

High-speed flight presents a variety of challenges for state estimation. If we are interested in light weight and low inertia solutions, cameras and IMUs are the best sensors. Recent research in vision-based navigation by our group [16] has demonstrated robust algorithms in feature-rich environments. The algorithms use a monocular camera with image features being tracked across time fused with measurements from an IMU to get an estimate of the motion of the robot. In the tests reported in this paper we use the Semi-direct monocular Visual Odometry (SVO) algorithm [17. A disadvantage of these systems is the inability to obtain an estimate of scale from measurements obtain from a stationary starting configuration. Using stereo systems allow us to get the scale immediately [18. However, during high-speed flight, two major challenges manifest themselves. The first challenge involves the attitude estimator must incorporate models of drag and large accelerations when using measurements from the IMU. Second, it is difficult to achieve robustness with feature tracking during high speeds, especially with high pitch and roll rates because of motion blur, particularly in poor illumination.

\section{Nonlinear Controllers}

Sustained high speed flight involving large attitude changes poses challenges for non only for motion estimation but also for control. We use nonlinear controllers 19 with feed-forward control inputs enabling us to handle large deviations from hover for aggressive maneuvers.

\section{Dense Mapping}

Building maps for motion planning at high speeds with light weight platforms poses several challenges. First, to build dense maps over the time scale of $10 \mathrm{~m}$ (see Fig. 3) at 30 Hz. using cameras we need computational resources that significantly exceed what is available on standard, light-weight CPUs. The alternative is to use an active imaging system such as a 3-D lidar, which increases the weight substantially. As a compromise, we used a 2-D laser scanner mounted on a horizontal servo to enable mapping of 3-D obstacles (see Fig. 4). Second, the process of building a 3-D global map for motion planning is also computationally intensive. Instead, we create a body-centric representation of the occupancy around the vehicle and represent the free space as a union of convex polyhedra. We are able to generate smooth motion plans in 
free space at a minimum of $30 \mathrm{~Hz}$.

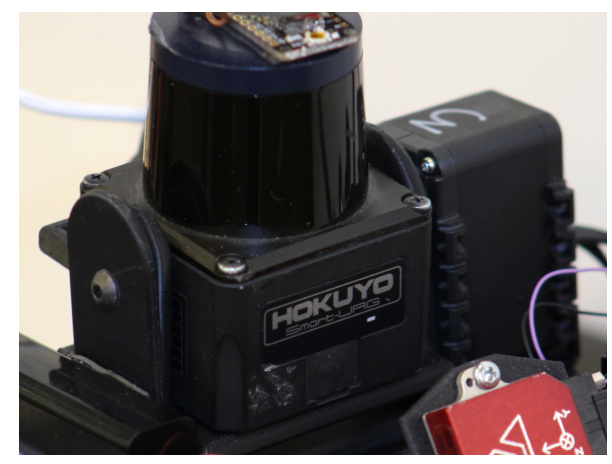

Figure 4: The 2-D nodding laser scanner represents a compromise between the heavier 3-D lidar and the lighter cameras which do not lend themselves to dense mapping.

\section{SYSTEM DESIGN}

The FALCON is largely based on commercially available off the shelf (COTS) components. We chose the DJI 20] F450 frame and E600 motors with 12in propellers to obtain the maximum thrust to weight ratio with a compact footprint and the Pixhawk 21] autopilot for attitude estimation and control. Two Point Grey Chameleon3 cameras synchronized with a VectorNav VN-100 IMU enable state estimation, in addition to a Hokuyo UST-20LX LiDAR on a custom built nodding head gimbal. Additionally, the FALCON includes a downward facing sensor module consisting of a BlueFOX MLC200w camera and a LidarLite laser altimeter. The primary CPU is an Intel NUC with a $3.1 \mathrm{GHz}$ i7 processor with $16 \mathrm{~Gb}$ of RAM and a $120 \mathrm{~Gb}$ solid state hard drive. Communication with the robot for monitoring experiments takes place via an onboard high power, long range $802.11 \mathrm{~b} / \mathrm{g} / \mathrm{n}$ WiFi transceiver. A custom designed Dragonfly power distribution board provides power conditioning, DC-DC converters, power connectors, and battery monitor. Finally, a 6S 4000mAh lithium polymer battery provides the base platform (without sensors) a thrust to weight ratio of 5 , with a 15 minute flight time. Fully loaded, the weight with all components is $3.0 \mathrm{~kg}$ with a thrust-weight ratio of 2.5 . This thrust to weight ratio allows the robot to reach maximum speeds of $20 \mathrm{~m} / \mathrm{s}$ with a maximum acceleration of $6 \mathrm{~m} / \mathrm{s}^{2}$.

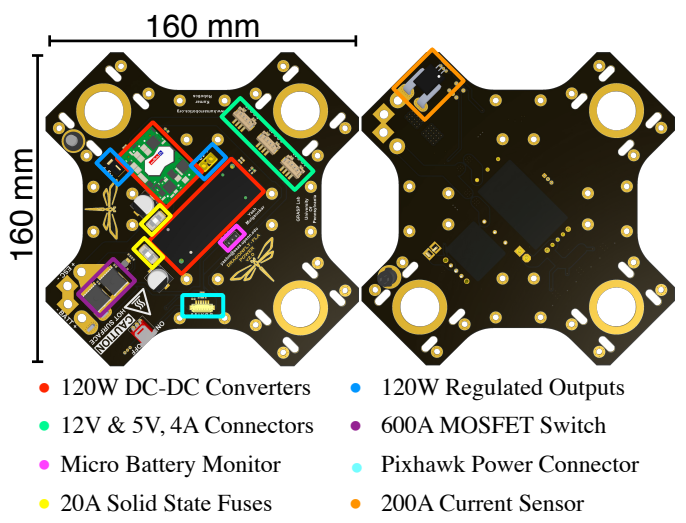

Figure 5: Dragonfly Power Distribution Board
In the "Dense Mapping" Section above, we briefly discussed the trade-offs between choosing 3-D lidars and cameras to minimize the total weight. A major consideration in choosing the present configuration with a nodding 2-D lidar instead of a camera-only configuration was the lack of light weight processors. It is worth noting that new tools are coming online that point to promising avenues for development. As seen in Table 1, new processing units are approaching ratios that exceed 160 MFlops/gram, which is nearly $10 \mathrm{x}$ processors available only 2 years ago.

As shown in Figure 6 the sensors and processors (along with cabling) contribute to $45 \%$ of the mass of the vehicle. This weight can be substantially reduced by integrating the sensors and processors into light weight packages. Using lighter weight composites will further reduce the total mass of the frame and the cage which contribute nearly $20 \%$ of the vehicle. It is unlikely that the Lithium polymer batteries or alternative powers will improve substantially in terms of either specific power or specific weight in the near future, even though advances are being made in terms of energy per unit volume. Finally, because quadrotors consume approximately 200 Watts/kg., as shown in Figure 7 nearly $90 \%$ of the power consumption arises from the weight of the vehicle. Thus light weight computing / sensing is more important than low power computing/sensing.

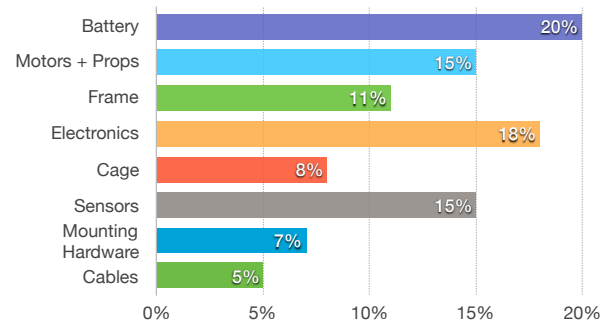

Figure 6: Mass distribution of the Falcon UAV

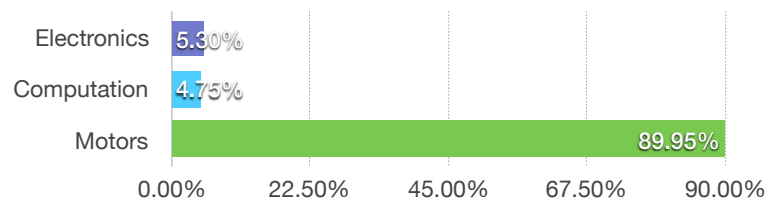

Figure 7: Power consumption at hover

\section{CONTACT}

*Yash Mulgaonkar, yashm@grasp.upenn.edu

\section{REFERENCES}

[1] Christian Bermes. Design and dynamic modeling of autonomous coaxial micro helicopters. PhD thesis, Diss., Eidgenössische Technische Hochschule ETH Zürich, Nr. 18847, 2010, 2010.

[2] Samir Bouabdallah. Design and control of quadrotors with application to autonomous flying. $\mathrm{PhD}$ thesis, Ecole Polytechnique Federale de Lausanne, 2007. 
Table 1: Light weight versus low power computers for autonomous flight.

\begin{tabular}{|l|l|l|l|l|l|}
\hline \multicolumn{1}{|c|}{ Computer } & $\begin{array}{l}\text { Intel NUC } \\
\text { (i3-5010U) }\end{array}$ & $\begin{array}{l}\text { Intel NUC } \\
(\text { i5-5250U) }\end{array}$ & $\begin{array}{l}\text { Intel NUC } \\
(\text { i7-5557U) }\end{array}$ & $\begin{array}{l}\text { Odroid XU3 } \\
\text { (Exynos 5422) }\end{array}$ & $\begin{array}{l}\text { Qualcomm Eagle } \\
\text { (Snapdragon 801) }\end{array}$ \\
\hline Cores & 2 & 2 & 2 & 4 & 4 \\
\hline Clock Speed (GHz) & 1.7 & 2.1 & 3.1 & 2 & 2.5 \\
\hline MFLOPS (Single-Core) & 1900 & 2730 & 3440 & 1030 & 1200 \\
\hline MFLOPS (Multi-Core) & 4250 & 5400 & 7480 & 4270 & 4350 \\
\hline Mass (g) & 210 & 210 & 210 & 38 & 27 \\
\hline MFlops / g (Single-Core) & 9.05 & 13 & 16.38 & 27.11 & 44.44 \\
\hline MFlops / g Multi-Core) & 20.24 & 25.71 & 35.62 & 112.37 & 161.11 \\
\hline
\end{tabular}

[3] Darryll J Pines and Felipe Bohorquez. Challenges facing future micro-air-vehicle development. Journal of aircraft, 43(2):290-305, 2006.

[4] Ascending Technologies GmbH. http://www.asctec.de/.

[5] Sergei Lupashin, Angela Schöllig, Michael Sherback, and Raffaello D'Andrea. A simple learning strategy for high-speed quadrocopter multi-flips. In Robotics and Automation (ICRA), 2010 IEEE International Conference on, pages 1642-1648. IEEE, 2010.

[6] D. Mellinger and V. Kumar. Minimum snap trajectory generation and control for quadrotors. Robotics and $A u$ tomation (ICRA), 2011 IEEE International Conference on, pages 2520-2525, May 2011.

[7] N. Michael, S. Shen, K. Mohta, Y. Mulgaonkar, V. Kumar, K. Nagatani, Y. Okada, S. Kiribayashi, K. Otake, K. Yoshida, K. Ohno, E. Takeuchi, and S. Tadokoro. Collaborative mapping of an earthquakedamaged building via ground and aerial robots. Journal Field Robotics, 29(5):832-841, 2012.

[8] Q. Lindsey, D. Mellinger, and V. Kumar. Construction of cubic structures with quadrotor teams. Robotics Science and Systems, 2011.

[9] Matthew Turpin, Kartik Mohta, Nathan Michael, and Vijay Kumar. Goal assignment and trajectory planning for large teams of aerial robots. Robotics: Science and Systems (RSS), June 2013.

[10] A. Bachrach, A. de Winter, R. He, G. Hemann, S. Prentice, and N. Roy. Range - robust autonomous navigation in gps-denied environments. In Robotics and Automation (ICRA), 2010 IEEE International Conference on, pages 1096-1097, May 2010.

[11] Dimitrios G Kottas, Joel A Hesch, Sean L Bowman, and Stergios I Roumeliotis. On the consistency of visionaided inertial navigation. In Experimental Robotics, pages 303-317. Springer, 2013.

[12] Friedrich Fraundorfer, Lionel Heng, Dominik Honegger, Gim Hee Lee, Lorenz Meier, Petri Tanskanen, and Marc Pollefeys. Vision-based autonomous mapping and exploration using a quadrotor mav. In Intelligent Robots and
Systems (IROS), 2012 IEEE/RSJ International Conference on, pages 4557-4564. IEEE, 2012.

[13] Albert S Huang, Abraham Bachrach, Peter Henry, Michael Krainin, Daniel Maturana, Dieter Fox, and Nicholas Roy. Visual odometry and mapping for autonomous flight using an rgb-d camera. In International Symposium on Robotics Research (ISRR), volume 2, 2011.

[14] Yash Mulgaonkar, Michael Whitzer, Brian Morgan, Christopher M Kroninger, Aaron M Harrington, and Vijay Kumar. Power and weight considerations in small, agile quadrotors. In SPIE Defense+ Security, pages 90831Q-90831Q. International Society for Optics and Photonics, 2014.

[15] Vijay Kumar and Nathan Michael. Opportunities and challenges with autonomous micro aerial vehicles. The International Journal of Robotics Research, 31(11):1279-1291, 2012.

[16] G. Loianno, Y. Mulgaonkar, C. Brunner, D. Ahuja, A. Ramanandan, M. Chari, S. Diaz, and V. Kumar. Smartphones power flying robots. In Intelligent Robots and Systems (IROS), 2015 IEEE/RSJ International Conference on, pages 1256-1263, Sept 2015.

[17] Christian Forster, Matia Pizzoli, and Davide Scaramuzza. SVO: Fast semi-direct monocular visual odometry. In IEEE International Conference on Robotics and Automation (ICRA), 2014.

[18] S. Shen, Y. Mulgaonkar, N. Michael, and V. Kumar. Multi-sensor fusion for robust autonomous flight in indoor and outdoor environments with a rotorcraft mav. In 2014 IEEE International Conference on Robotics and Automation (ICRA), pages 4974-4981, May 2014.

[19] T. Lee, M. Leoky, and N. H. McClamroch. Geometric tracking control of a quadrotor uav on se(3). In 49th IEEE Conference on Decision and Control (CDC), pages 5420-5425, Dec 2010.

[20] DJI. http://www.dji.com/.

[21] Pixhawk Autopilot. https://pixhawk.org. 